THE MIND IN EXILE 



\title{
The Mind in Exile
}

THOMAS MANN IN PRINCETON

\author{
STA N LEY COR NGOLD
}

PRINCETON UNIVERSITY PRESS

PRINCETON \& OXFORD 


\section{Copyright $(\odot 2022$ by Stanley Corngold}

Princeton University Press is committed to the protection of copyright and the intellectual property our authors entrust to us. Copyright promotes the progress and integrity of knowledge. Thank you for supporting free speech and the global exchange of ideas by purchasing an authorized edition of this book. If you wish to reproduce or distribute any part of it in any form, please obtain permission.

Requests for permission to reproduce material from this work should be sent to permissions@press.princeton.edu

Published by Princeton University Press 41 William Street, Princeton, New Jersey o8540 6 Oxford Street, Woodstock, Oxfordshire OX20 ITR

press.princeton.edu

All Rights Reserved

Library of Congress Cataloging-in-Publication Data

Names: Corngold, Stanley, author.

Title: The mind in exile : Thomas Mann in Princeton / Stanley Corngold.

Description: Princeton : Princeton University Press, 2022. | Includes bibliographical references and index.

Identifiers: LCCN 2021018405 (print) | LCCN 2021018406 (ebook) | ISBN 9780691201641 (hardback) | ISBN 9780691229676 (ebook)

Subjects: LCSH: Mann, Thomas, 1875-1955 - Exile—United States. | Mann, Thomas, 1875-1955-Political and social views. | Mann, Thomas, 1875-1955-Homes and haunts—New Jersey—Princeton | Mann, Thomas, 1875-1955—Friends and associates. | Princeton (N.J.) — Intellectual life. | Authors, German2oth century-Biography.

Classification: LCC PT2625.A44 Z544197 2022 (print) | LCC PT2625.A44 (ebook) | DDC $833 / .912[\mathrm{~B}]$ - dc23

LC record available at https://lccn.loc.gov/2021018405

LC ebook record available at https://lccn.loc.gov/2021018406

British Library Cataloging-in-Publication Data is available

Editorial: Rob Tempio, Matt Rohal, and Chloe Coy

Production Editorial: Mark Bellis

Jacket Design: Layla Mac Rory

Production: Erin Suydam

Publicity: Alyssa Sanford and Carmen Jimenez

Copyeditor: Jodi Beder

Jacket photo by Fred Stein $($ fredstein.com

This book has been composed in Arno

Printed on acid-free paper. $\infty$

Printed in the United States of America

$\begin{array}{llllllllll}10 & 9 & 8 & 7 & 6 & 5 & 4 & 3 & 2 & 1\end{array}$ 